\title{
Natural Doundary Element Method for Elliptic Boundary Value Problems in Domains with Concave Angle
}

\author{
Qi-Kui Du \\ School of Mathematics and Computer Science, Nanjing Normal University, Nanjing 210097, P R China. \\ De-Hao Yu \\ Institute of Computational Mathematics and Scientific/Engineering Computing, Academy of Mathematics and \\ System Science, Chinese Academy of Sciences, Beijing 100080, P R China.
}

Follow this and additional works at: https://jmstt.ntou.edu.tw/journal

Part of the Electrical and Computer Engineering Commons

\section{Recommended Citation}

Du, Qi-Kui and Yu, De-Hao (2001) "Natural Doundary Element Method for Elliptic Boundary Value Problems in Domains with Concave Angle," Journal of Marine Science and Technology. Vol. 9: Iss. 2, Article 2.

DOI: 10.51400/2709-6998.2437

Available at: https://jmstt.ntou.edu.tw/journal/vol9/iss2/2

This Research Article is brought to you for free and open access by Journal of Marine Science and Technology. It has been accepted for inclusion in Journal of Marine Science and Technology by an authorized editor of Journal of Marine Science and Technology. 


\section{Natural Doundary Element Method for Elliptic Boundary Value Problems in Domains with Concave Angle}

\section{Acknowledgements}

The authors first wish to thank the referees for many valuable sugguestions. Second, this work is partly supported by the Special Funds for State Major Basic Research Projects of China, Grant number 1999032800, and by the knowledge innovation program of the Chinese Academy Sciences. 


\title{
NATURAL BOUNDARY ELEMENT METHOD FOR ELLIPTIC BOUNDARY VALUE PROBLEMS IN DOMAINS WITH CONCAVE ANGLE
}

\author{
Qi-Kui Du* and De-Hao Yu**
}

Keywords: natural boundary reduction (NBR), concave angle domain, numerical implementation, coupling.

\section{ABSTRACT}

In this paper the natural boundary reduction for some elliptic boundary value problems with concave angle domains and its natural boundary element methods are investigated. Natural integral equations and Poisson integral formulae are given. A finite element methods of natural integral equations are discussed in details. The convergence of approximate solutions and their error estimates are obtained. Some numerical experiments are presented to demonstrate the performance of the method and our estimates. As an application, we present the coupling of FEM and natural boundary element.

\section{INTRODUCTION}

In this paper we consider a kind of elliptic problems with concave angle domains in two dimensions, which is Neumann boundary-value problem. Let $\Omega$ and $\Omega^{c}$ are a bounded sector domain with angle $\alpha$ and an exterior concave angle domains, respectively, and $0<\alpha$ $\leq 2 \pi$.

We consider some linear elliptic second-order boundary value problems in two dimensions. The boundary of domain $\Omega$ or $\Omega^{c}$ is decomposed into three disjoint parts, $\Gamma, \Gamma_{0}$ and $\Gamma_{\alpha} . \quad \Gamma, \Gamma_{0}$ and $\Gamma_{\alpha}$ where a Neumann boundary conditions are given. The statement of the problems considered is:

$$
\begin{array}{ll}
\Delta u+\beta u=0, & \text { in } \Omega \text { or } \Omega^{c} \\
\frac{\partial u}{\partial n}(r, 0)=0, & \text { on } \Gamma_{0}
\end{array}
$$

Paper Received June 28, 2001. Author for Correspondence: Qi-Kui Du. *School of Mathematics and Computer Science, Nanjing Normal University, Nanjing 210097, P R China.

**Institute of Computational Mathematics and Scientific/Engineering Computing, Academy of Mathematics and System Science, Chinese Academy of Sciences, Beijing 100080, P R China.

$$
\begin{aligned}
& \frac{\partial u}{\partial n}(r, \alpha)=0, \quad \text { on } \Gamma_{\alpha} \\
& \frac{\partial u}{\partial n}(R, \theta)=g_{n}(R, \theta), \quad \text { on } \Gamma
\end{aligned}
$$

If domain is $\Omega^{c}$, some conditions at infinity.

Here, $u$ is the unknown function, $\frac{\partial u}{\partial n}$ is the normal derivative of $u$ on boundary, $g_{n}(R, \theta)$ is given function, and $\beta$ is a constant. Equation (1) is Laplace, Helmholtz or modified Helmholtz equation, according as $\beta$ is zero, positive or negative. In this paper we shall concentrate on the method of natural boundary element method [1, $2,3,4]$ or DtN method [5, 6]. It is well-known that the natural integral equation is hypersingular, its kernel function has non-integrable singularity, the integral is to be understood in the sense of the theory of distribution. It can be referred to [10]. The condition at infinity (5) is as follows. If $\beta \leq 0$ then the solution $u$ is required to vanish at infinity; If $\beta>0$ then at infinity the solution $u$ is required to be imposed a radication condition

$$
\lim _{r \rightarrow+\infty} \sqrt{r}\left(\frac{\partial u}{\partial r}-\sqrt{\beta} u\right)=0
$$

In the above problems, $\mathrm{Yu}$ [1] has only investigated the problems for $\beta=0$. However, there are some more significant engineering background for $\beta \neq 0$, such as waveguide, electromagnetic radiation, geophysics, meteorology, etc. In addition, discretization in time for some time-dependent problems is reduced to the model (1) [7]. So, it has been of great importance in theory and practical applications to investigate some numerical methods of the above problems systematically.

Givoli, Rivkin and Keller [11], Wu and Han [12] used a method called the DtN finite-element method to some elliptic boundary value problems in domains with corners and singularities. They gave a sequence of approximations to the exact boundary conditions on an artificial boundary by using Green's function. On the boundary, the Neumann boundary condition is related to the Dirchlet boundary condition, or the second-order 
derivative of the solution on the artificial boundary. Bao and Han [13] gave a high-order local approximate artificial boundary condition. Accurate numerical results were obtained for this approach in $[11,12]$. In this paper, we follow the idea of $[2,5,10]$ for solving some elliptic boundary value problems in domains (bounded and unbounded) with concave angle, and give their natural boundary reduction and natural boundary element method (or called the $D t N$ finite-element method).

The outline of the paper is as follows. In Section 2 we derive natural integral equations on $\Gamma$ and Poisson integral equations in $\Omega$ or $\Omega^{c}$. We consider the Laplace, Helmholtz and modified Helmholtz equations in $\Omega$ or $\Omega^{c}$. In Section 3 we discuss the numerical implementations of natural integral equations by finite element method. Section 4 contains some error analyses for the finite element scheme. To demonstrate the performance of the method, we present in Section 5 some numerical experiments and examples. As an application, we also discuss the coupling of FEM and NBE in infinite sector domain in Section 6. We close with concluding remarks in Section 7.

\section{NATURAL BOUNDARY REDUCTION}

For problems (1)-(5), the function $g_{n}(R, \theta)$ satisfies compatibility condition $\int_{0}^{\alpha} g_{n}(R, \theta) d \theta=0$ if $\beta=0$.

\section{Bounded Sector Domain}

Domain $\Omega$, boundaries $\Gamma, \Gamma_{0}$ and $\Gamma_{\alpha}$ are as follows: $\Omega:=(r, \theta) \mid 0<r<R, \theta \in(0, \alpha)\}, \Gamma:=\{(R, \theta) \mid \theta \in(0$, $\alpha)\}, \Gamma_{0}:=\{(r, 0) \mid 0 \leq r \leq R\}, \Gamma_{\alpha}:=\{(r, \alpha) \mid 0 \leq r \leq R\}$.

By separation of variables we find the general solution which satisfies (1) in the domain $\Omega$ and the conditions mentioned in section 1 :

$$
u(r, \theta)=\sum_{n=0}^{+\infty} A_{n} \cdot B_{n}(r) \cos \frac{n \pi \theta}{\alpha}, \quad r<R
$$

The coefficients $B_{n}(r)$ for all different cases of $\beta$ considered are summarized in Table 1. There $I_{v}(x)$ is the modified Bessel function of the first kind of order $\mathrm{v}$, and $J_{v}(x)$ is the Bessel function of order $v[8,9]$, i.e.,

$$
\begin{aligned}
& I_{v}(x)=\sum_{j=0}^{+\infty} \frac{1}{j ! \cdot \Gamma(j+1+v)}\left(\frac{x}{2}\right)^{2 j+v}, \\
& J_{v}(x)=\sum_{j=0}^{+\infty}(-1)^{j} \frac{1}{j ! \cdot \Gamma(j+1+v)}\left(\frac{x}{2}\right)^{2 j+v},
\end{aligned}
$$

Table 1. The coefficients $\boldsymbol{B}_{n}(r)$ for all different cases of $\beta$

\begin{tabular}{cccc}
\hline Parameter & $\beta<0$ & $\beta=0$ & $\beta>0$ \\
\hline $\mathrm{B}_{\mathrm{n}}(\mathrm{r})$ & $I_{\frac{n \pi}{\alpha}}(\sqrt{|\beta|} r)$ & $r^{\frac{n \pi}{\alpha}}$ & $J_{\frac{n \pi}{\alpha}}(\sqrt{\beta} r)$ \\
\hline
\end{tabular}

From the following

$$
\begin{aligned}
u(R, \theta) & =\lim _{r \rightarrow R^{-}} u(r, \theta) \\
& =\sum_{n=0}^{+\infty} A_{n} B_{n}(R) \cos \frac{n \pi \theta}{\alpha}
\end{aligned}
$$

Using the orthogonality of the cosines, we obtain the coefficients $A_{n}, n=0,1,2, \ldots$,

$$
A_{n}=\frac{1}{\alpha} \varepsilon_{n} B_{n}(R)^{-1} \int_{0}^{\alpha} u(R, \theta) \cos \frac{n \pi \theta}{\alpha} d \theta,
$$

Hence

$$
\begin{gathered}
u(r, \theta)=\frac{1}{\alpha} \sum_{n=0}^{+\infty} \varepsilon_{n} B_{n}(r) \cdot B_{n}(R)^{-1} \int_{0}^{\alpha} u\left(R, \theta^{\prime}\right) \\
\cdot \cos \frac{n \pi \theta}{\alpha} \cdot \cos \frac{n \pi \theta^{\prime}}{\alpha} d \theta^{\prime}, \quad r<R
\end{gathered}
$$

Where $R$ is the radius of circular arc $\Gamma$. If we let

$$
G_{n}(r ; R):=B_{n}(r) \cdot R_{n}(R)^{-1}
$$

then (10) can be expressed as follows:

$$
\begin{aligned}
u(r, \theta) & =\frac{1}{\alpha} \sum_{n=0}^{+\infty} \varepsilon_{n} G_{n}(r ; R) \int_{0}^{\alpha} u\left(R, \theta^{\prime}\right) \\
& \cdot \cos \frac{n \pi \theta}{\alpha} \cdot \cos \frac{n \pi \theta^{\prime}}{\alpha} d \theta^{\prime}, \\
& \equiv \mathcal{P} u(R, \theta), \quad r<R
\end{aligned}
$$

We differentiate (11) with respect to $r$ and take the limit as $r$ approaches to $R^{-}$, to obtain

$$
\begin{aligned}
\frac{\partial u}{\partial r} & =\frac{\pi}{R \alpha^{2}} \sum_{n=0}^{+\infty} \varepsilon_{n} Z_{n} \int_{0}^{\alpha} u\left(R, \theta^{\prime}\right) \\
& \cdot \cos \frac{n \pi \theta}{\alpha} \cdot \cos \frac{n \pi \theta^{\prime}}{\alpha} d \theta^{\prime}
\end{aligned}
$$

The coefficients $G_{n}(r ; R)$ and $Z_{n}$ in (11) and (12) are summarized in Table 2 . And $\varepsilon_{n}$ is: $\varepsilon_{n}=1$, if $n=0$. $\varepsilon_{n}=2$, if $n \neq 0$.

Table 2. The coefficients $G_{n}(r ; R)$ and $Z_{n}$ for all different cases of $\beta$

\begin{tabular}{ccc}
\hline$\beta$ & $G_{n}(r ; R)$ & $Z_{n}$ \\
\hline$<0$ & $\frac{I_{\frac{n \pi}{\alpha}}(\sqrt{|\beta|} r)}{I_{\frac{n \pi}{\alpha}}(\sqrt{|\beta|} R)}$ & $\frac{\alpha \sqrt{|\beta|} R}{\pi} \cdot \frac{I_{\frac{n \pi}{\alpha}}^{\prime}(\sqrt{|\beta|} R)}{I_{\frac{n \pi}{\alpha}}(\sqrt{|\beta|} R)}$ \\
$=0$ & $\left(\frac{r}{R}\right)^{\frac{n \pi}{\alpha}}$ & $n$ \\
$>0$ & $\frac{J_{\frac{n \pi}{\alpha}}(\sqrt{\beta} r)}{J_{\frac{n \pi}{\alpha}}(\sqrt{\beta R})}$ & $\frac{\alpha \sqrt{|\beta|} R}{\pi} \cdot \frac{J_{\frac{n \pi}{\alpha}}^{\prime}(\sqrt{\beta R} R)}{J_{\frac{n \pi}{\alpha}}(\sqrt{\beta R})}$ \\
\hline
\end{tabular}




\section{Infinite Sector Domain}

Domain $\Omega^{c}$, boundaries $\Gamma, \Gamma_{0}$ and $\Gamma_{\alpha}$ are as follows: $\Omega^{c}:=\{(r, \theta) \mid r>R, \theta \in(0, \alpha)\}, \Gamma:=\{(R, \theta) \mid \theta \in(0$, $\alpha)\}, \Gamma_{0}:=\{(r, 0) \mid r>R\}, \Gamma_{\alpha}:=\{(r, \alpha) \mid r>R\}$.

Similar to the above methods, we get the solution of the problem (1)-(5) for all cases of $\beta$ considered

$$
\begin{aligned}
u(r, \theta) & =\frac{1}{\alpha} \sum_{n=0}^{+\infty} \varepsilon_{n} G_{n}(r ; R) \int_{0}^{\alpha} u\left(R, \theta^{\prime}\right) \\
& \cdot \cos \frac{n \pi \theta}{\alpha} \cdot \cos \frac{n \pi \theta^{\prime}}{\alpha} d \theta^{\prime} \\
& \equiv \mathcal{P} u(R, \theta), \quad r>R
\end{aligned}
$$

Furthermore

$$
\begin{aligned}
\frac{\partial u}{\partial r}= & -\frac{\pi}{R \alpha^{2}} \sum_{n=0}^{+\infty} \varepsilon_{n} Z_{n} \int_{0}^{\alpha} u\left(R, \theta^{\prime}\right) \\
& \cdot \cos \frac{n \pi \theta}{\alpha} \cdot \cos \frac{n \pi \theta^{\prime}}{\alpha} d \theta^{\prime}, \quad \text { on } \Gamma
\end{aligned}
$$

The coefficients $G_{n}(r ; R)$ and $Z_{n}$ in (13) and (14) are summarized in Table 3 . There $K_{v}(x)$ is the modified Bessel function of the second kind of order $v$, and $H_{v}^{(1)}$ $(x)$ is the Hankel function of the first kind of order $v[8$, 9], i.e.,

$$
\begin{aligned}
& K_{v}(x)=\frac{\pi}{2} \cdot \frac{I_{-v}(x)-I_{v}(x)}{\sin v \pi}, \\
& H_{v}^{(1)}(x)=\frac{i}{\sin v \pi} \cdot\left[J_{v}(x) \cdot e^{-i v \pi}-J_{-v}(x)\right]
\end{aligned}
$$

Since we have known that $\frac{\partial}{\partial n}=\frac{\partial}{\partial r}$ for interior problem, and $\frac{\partial}{\partial n}=-\frac{\partial}{\partial r}$ for exterior problem, on $\Gamma$. Therefore (12) and (14) can be written as follows:

$$
\begin{aligned}
\frac{\partial u}{\partial n} & =\frac{\pi}{R \alpha^{2}} \sum_{n=0}^{+\infty} \varepsilon_{n} Z_{n} \int_{0}^{\alpha} u\left(R, \theta^{\prime}\right) \\
& \cdot \cos \frac{n \pi \theta}{\alpha} \cdot \cos \frac{n \pi \theta^{\prime}}{\alpha} d \theta^{\prime} \\
& \equiv \mathcal{K} u(R, \theta), \text { on } \Gamma
\end{aligned}
$$

Table 3. The coefficients $G_{n}(r ; R)$ and $Z_{n}$ for all different cases of $\beta$

\begin{tabular}{ccc}
\hline$\beta$ & $G_{n}(r ; R)$ & $Z_{n}$ \\
\hline$<0$ & $\frac{K_{\frac{n \pi}{\alpha}}(\sqrt{|\beta|} r)}{K_{\frac{n \pi}{\alpha}}(\sqrt{|\beta|} R)}$ & $\frac{\alpha \sqrt{|\beta|} R}{\pi} \cdot \frac{K_{\frac{n \pi}{\alpha}}^{\prime}(\sqrt{|\beta|} R)}{K_{\frac{n \pi}{\alpha}}(\sqrt{|\beta|} R)}$ \\
$=0$ & $\left(\frac{R}{r}\right)^{\frac{n \pi}{\alpha}}$ & $n$ \\
$>0$ & $\frac{H_{\frac{n \pi}{\alpha}}(\sqrt{\beta} r)}{H_{\frac{n \pi}{\alpha}}(\sqrt{\beta} R)}$ & $\frac{\alpha \sqrt{|\beta|} R}{\pi} \cdot \frac{H_{\frac{n \pi}{\alpha}}^{\prime}(\sqrt{\beta} R)}{H_{\frac{n \pi}{\alpha}}(\sqrt{\beta} R)}$ \\
\hline
\end{tabular}

The coefficients $Z_{n}$ in (15) are given by Tables 2 and 3 , respectively. That is, the coefficients $Z_{n}$ are given in Table 2 for the problem in bounded sector domain, and the coefficients $Z_{n}$ are given in Table 3 for the problem in infinite sector domain. Equations (11) and (13) are often called the Poisson integral formulae, and equation (15) is called natural integral equation [10] or Dirichlet-to-Neumann $(D t N)$ map [8, 9]. In practice, the natural integral equation is truncated after a finite number of terms, $M$, namely,

$$
\begin{aligned}
\frac{\partial u}{\partial n} & =\frac{\pi}{R \alpha^{2}} \sum_{n=0}^{M} \varepsilon_{n} Z_{n} \int_{0}^{\alpha} u\left(R, \theta^{\prime}\right) \\
& \cdot \cos \frac{n \pi \theta}{\alpha} \cdot \cos \frac{n \pi \theta^{\prime}}{\alpha} d \theta^{\prime} \\
& \equiv \mathcal{K}_{M} u(R, \theta),
\end{aligned}
$$

To cope with the numerical analysis, referring to [10] we recall an equivalent definition of Sobolev space $H^{s}(\Gamma)$ for any real number $s$ :

$$
\begin{aligned}
& \forall f \in H^{s}(\Gamma) \Leftrightarrow \\
& f(R, \theta)=\sum_{n=-\infty}^{+\infty} \varepsilon_{n} f_{n} \cdot\left(e^{i \frac{n \pi}{\alpha} \theta}+e^{-i \frac{n \pi}{\alpha} \theta}\right) \\
& \text { and } \sum_{n=-\infty}^{+\infty}\left[1+\left(\frac{n \pi}{\alpha}\right)^{2}\right]^{s} \cdot\left|\varepsilon_{n} f_{n}\right|^{2}<+\infty
\end{aligned}
$$

where $\Gamma:=\{(R, \theta): 0<\theta \leq 2 \pi, R>0\}$, and $f_{n}=$ $\frac{1}{2 \alpha} \int_{0}^{\alpha} f(R, \theta) \cdot\left(e^{i \frac{n \pi}{\alpha} \theta}+e^{-i \frac{n \pi}{\alpha} \theta}\right) d \theta$. Thus we assign the following norm on $H^{s}(\Gamma)$ : For any $f \in H^{s}(\Gamma)$

$$
\|f(R, \theta)\|_{s, \Gamma}^{2}:=\sum_{n=-\infty}^{+\infty}\left[1+\left(\frac{n \pi}{\alpha}\right)^{2}\right]^{s} \cdot\left|\varepsilon_{n} f_{n}\right|^{2}
$$

Especially, if $s=0$ we have

$$
\|f(R, \theta)\|_{0, \Gamma}:=\left[\sum_{n=-\infty}^{+\infty}\left|\varepsilon_{n} f_{n}\right|^{2}\right]^{\frac{1}{2}}=\|f(R, \theta)\|_{L^{2}(\Gamma)} .
$$

In order to construct the weak form of problem (15), we define the followings $(d s=R d \theta)$ :

$$
\begin{aligned}
\hat{D}\left(u_{0}, v_{0}\right): & =\left\langle\mathcal{K} u_{0}(R, \theta), v_{0}>_{\Gamma}\right. \\
\equiv & \int_{\Gamma} \mathcal{K} u_{0}(R, \theta) \cdot v_{0} d S \\
= & \frac{\pi}{\alpha^{2}} \sum_{n=0}^{+\infty} \varepsilon_{n} Z_{n} \int_{0}^{\alpha} \int_{0}^{\alpha} u_{0}\left(R, \theta^{\prime}\right) \\
& \cdot v_{0}(R, \theta) \cos \frac{n \pi \theta}{\alpha} \cos \frac{n \pi \theta^{\prime}}{\alpha} d \theta^{\prime} d \theta, \\
\hat{F}_{n}\left(v_{0}\right):= & \int_{\Gamma} \frac{\partial u}{\partial n} \cdot v_{0}(R, \theta) d S \\
\equiv & R \int_{0}^{\alpha} g_{n}(R, \theta) \cdot v_{0}(R, \theta) d \theta
\end{aligned}
$$


Then the weak form of (15) is:

(P) Find $u_{0} \in H^{\frac{1}{2}}(\Gamma)$ such that

$$
\hat{D}\left(u_{0}, v_{0}\right)=\hat{F}_{n}\left(v_{0}\right), \quad \forall v_{0} \in H^{\frac{1}{2}}(\Gamma)
$$

Lemma 1. If $u=\sum_{n=-\infty}^{+\infty} u_{n}\left(e^{i \frac{n \pi}{\alpha} \theta}+e^{-i \frac{n \pi}{\alpha} \theta}\right), v=\sum_{n=-\infty}^{+\infty} u_{n}$ $\left(e^{i \frac{n \pi}{\alpha} \theta}+e^{-i \frac{n \pi}{\alpha} \theta}\right)$, then

$$
\begin{aligned}
u^{*} v & =\int_{0}^{\alpha} u\left(R, \theta-\theta^{\prime}\right) \cdot \int_{0}^{\alpha}\left(R, \theta^{\prime}\right) d \theta^{\prime} \\
& =\sum_{n=-\infty}^{+\infty}\left(2 \alpha u_{n} \cdot v_{n}\right)\left(e^{i \frac{n \pi}{\alpha} \theta}+e^{-i \frac{n \pi}{\alpha} \theta}\right)
\end{aligned}
$$

Proof It is not difficult to obtain that the following

$$
\begin{aligned}
& \int_{0}^{\alpha}\left(e^{-i \frac{n \pi}{\alpha} \theta}+e^{i \frac{n \pi}{\alpha} \theta}\right) \cdot\left(e^{i \frac{m \pi}{\alpha} \theta}+e^{-i \frac{m \pi}{\alpha} \theta}\right) d \theta \\
& = \begin{cases}2 \alpha, & m= \pm n \\
0, & \text { otherwise }\end{cases}
\end{aligned}
$$

Thus

$$
\begin{aligned}
u^{*} v & =\int_{0}^{\alpha} u\left(R, \theta-\theta^{\prime}\right) \cdot v\left(R, \theta^{\prime}\right) d \theta^{\prime} \\
& =\sum_{n=-\infty}^{+\infty} u_{n} e^{i \frac{n \pi}{\alpha} \theta} \sum_{n=-\infty}^{+\infty} u_{m} \int_{0}^{\alpha}\left(e^{-i \frac{n \pi}{\alpha} \theta}\right. \\
& \left.+e^{i \frac{n \pi}{\alpha} \theta}\right)\left(e^{i \frac{m \pi}{\alpha} \theta}+e^{-i \frac{m \pi}{\alpha} \theta}\right) d \theta \\
& =\sum_{n=-\infty}^{+\infty}\left(2 \alpha u_{n} v_{n}\right)\left(e^{i \frac{n \pi}{\alpha} \theta}+e^{-i \frac{n \pi}{\alpha} \theta}\right)
\end{aligned}
$$

This completes the proof.

From Lemma 1, we have

$$
\begin{aligned}
& \mathcal{K} u_{0}(R, \theta)=\frac{\pi}{R \alpha} \sum_{n=-\infty}^{+\infty} \varepsilon_{n} Z_{n} u_{n} \cdot\left(e^{i \frac{n \pi}{\alpha} \theta}+e^{-i \frac{n \pi}{\alpha} \theta}\right) \\
& \text { where } u_{n}=\frac{1}{2 \alpha} \int_{0}^{\alpha} u_{0}(R, \theta) \cdot\left(e^{i \frac{n \pi}{\alpha} \theta}+e^{-i \frac{n \pi}{\alpha} \theta}\right) d \theta .
\end{aligned}
$$

Theorem 1. For all non-negative real number $s$, natural integral operator $\mathcal{K}$ is a continuous linear operator from $H^{s+\frac{1}{2}}(\Gamma)$ to $H^{s-\frac{1}{2}}(\Gamma)$. In other words, there exists a positive constant $M_{1}$ such that for any $f \in H^{s+\frac{1}{2}}(\Gamma)$

$$
\|\mathcal{K} f\|_{s-\frac{1}{2}, \Gamma} \leq M_{1}\|f\|_{s-\frac{1}{2}, \Gamma}
$$

Proof By (20) and the definition of norm on $H^{s}(\Gamma)$, for any $f \in H^{s+\frac{1}{2}}(\Gamma)$ we have,

$$
\|\mathcal{L} f\|_{s-\frac{1}{2}, \Gamma}^{2}=\sum_{n=-\infty}^{+\infty}\left[1+\left(\frac{n \pi}{\alpha}\right)^{2}\right]^{s-\frac{1}{2}}\left|\frac{\pi}{R \alpha} \varepsilon_{n} Z_{n} f_{n}\right|^{2}
$$

$$
\begin{aligned}
& =\left(\frac{\pi}{R \alpha}\right)^{2} \sum_{n=-\infty}^{+\infty}\left[1+\left(\frac{n \pi}{\alpha}\right)^{2}\right]^{s+\frac{1}{2}}\left|\varepsilon_{n} f_{n}\right|^{2} \\
& \cdot\left(\left|Z_{n}\right|^{2} \cdot \frac{1}{1+\left(\frac{n \pi}{\alpha}\right)^{2}}\right) \\
& \leq C \cdot\left(\frac{\pi}{R \alpha}\right)^{2} \sum_{n=-\infty}^{+\infty}\left[1+\left(\frac{n \pi}{\alpha}\right)^{2}\right]^{s-\frac{1}{2}}\left|\varepsilon_{n} f_{n}\right|^{2} \\
& \leq C(\alpha, \beta) \cdot\|f\|_{s+\frac{1}{2}, \Gamma}^{2}
\end{aligned}
$$

Which completes the proof of Theorem 1.

Theorem 2. The bilinear form $\hat{D}(\bullet, \bullet)$ is symmetric and continuous on $H^{\frac{1}{2}}(\Gamma) \times H^{\frac{1}{2}}(\Gamma)$, and $H^{\frac{1}{2}}(\Gamma)$ coercive in the sense that exist two positive constants $M_{2}$ and $M_{3}$ such that for any $u, v \in H^{\frac{1}{2}}(\Gamma)$

$$
\begin{aligned}
& \hat{D}(u, v)=\hat{D}(v, u), \\
& \hat{D}(u, v) \leq M_{2} \cdot\|u\|_{\frac{1}{2}, \Gamma} \cdot\|v\|_{\frac{1}{2}, \Gamma}, \\
& \hat{D}(u, u) \geq M_{3} \cdot\|v\|_{\frac{1}{2}, \Gamma}^{2},
\end{aligned}
$$

Proof We have for any $u, v \in H^{\frac{1}{2}}(\Gamma)$

$$
\begin{aligned}
\hat{D}(u, v) & =\left\langle\mathcal{K} u, v>\equiv \int_{\Gamma} \mathcal{K} u \cdot v d S\right. \\
& =\int_{0}^{\alpha}\left(\frac{\pi}{R \alpha} \sum_{n=-\infty}^{+\infty} \varepsilon_{n} Z_{n} u_{n}\left(e^{i \frac{n \pi}{\alpha} \theta}+e^{-i \frac{n \pi}{\alpha} \theta}\right)\right) \\
& \cdot\left(\sum_{n=-\infty}^{+\infty} v_{m}\left(e^{i \frac{n \pi}{\alpha} \theta}+e^{-i \frac{n \pi}{\alpha} \theta}\right)\right) R d \theta \\
& =\frac{\pi}{\alpha} \int_{0}^{\alpha}\left(\sum_{n=-\infty}^{+\infty} \varepsilon_{n} Z_{n} u_{n}\left(e^{i \frac{n \pi}{\alpha} \theta}+e^{-i \frac{n \pi}{\alpha} \theta}\right)\right) \\
& \cdot\left(\sum_{n=-\infty}^{+\infty} v_{m}\left(e^{i \frac{m \pi}{\alpha} \theta}+e^{-i \frac{m \pi}{\alpha} \theta}\right)\right) d \theta \\
& =\frac{\pi}{\alpha} \sum_{n=-\infty}^{+\infty}\left(2 \alpha \varepsilon_{n} Z_{n} u_{n} v_{n}\right) \\
& =2 \pi \sum_{n=-\infty}^{+\infty} \varepsilon_{n} Z_{n} u_{n} v_{n}
\end{aligned}
$$

Hence, from this and Theorem 1 we have

$\hat{D}(u, v)=2 \pi \sum_{n=-\infty}^{+\infty} \varepsilon_{n} Z_{n} u_{n} v_{n}=\hat{D}(v, u)$

$\hat{D}(u, v)=\langle\mathcal{K} u, v\rangle$

$$
\leq\|\mathcal{K} u\|_{-\frac{1}{2}, \Gamma} \cdot\|v\|_{\frac{1}{2}, \Gamma}
$$




$$
\begin{aligned}
& \leq C \cdot\|u\|_{-\frac{1}{2}, \Gamma} \cdot\|v\|_{\frac{1}{2}, \Gamma} \\
\hat{D}(u, v) & =2 \pi \sum_{n=-\infty}^{+\infty}\left[1+\left(\frac{n \pi}{\alpha}\right)^{2}\right]^{-\frac{1}{2}}\left|\varepsilon_{n} u_{n}\right|^{2} \\
& \cdot\left(Z_{n} \cdot \frac{1}{\sqrt{1+\left(\frac{n \pi}{\alpha}\right)^{2}}}\right) \\
& \geq C \sum_{n=-\infty}^{+\infty}\left[1+\left(\frac{n \pi}{\alpha}\right)^{2}\right]^{-\frac{1}{2}}\left|\varepsilon_{n} u_{n}\right|^{2} \\
& =C\|u\|_{\frac{1}{2}, \Gamma}^{2}
\end{aligned}
$$

This completes the proof.

It follows immediately from the above Theorem 2, Corollary 1. The variational problem (18) has is wellposed on $H^{\frac{1}{2}}(\Gamma)$ when $\beta \neq 0$, and the variational problem (18) well-posed on $H^{\frac{1}{2}}(\Gamma) / \mathcal{P}_{0}$ when $\beta=0$ (where $\mathcal{P}_{0}$ is the polynomial of zero degree).

Corollary 1 implies immediately the wellposedness of the original problem (1)-(5).

\section{FINITE ELEMENT DISCRETIZATION}

Now we consider the approximation of the problem $(\mathrm{P})$. To the end, we discrete the circular arc $\Gamma$ into a finite number of element domains and let $S^{h}$ be the finite element subspace of space $H^{\frac{1}{2}}(\Gamma)$ corresponding to the subdivision and spanned by some type of shape functions, that is $S^{h} \subset H^{\frac{1}{2}}(\Gamma)$, then the discrete problem of variational problem (19):

$\left(P_{h}\right)$ Find $u_{0}^{h} \in S^{h}$ such that

$$
\hat{D}\left(u_{0}^{h}, v_{0}^{h}\right)=\hat{F}_{n}\left(u_{0}^{h}\right), \quad \forall v_{0}^{h} \in S^{h}
$$

\section{Linear Element}

We now partition the boundary $\Gamma$ into $N$ parts, the points of division are $\theta_{i}, i=1,2, \ldots, N-1$, i.e., $0 \equiv \theta_{0}<$ $\theta_{1}<\theta_{2} \ldots<\theta_{i}<\theta_{i+1}<\ldots<\theta_{N-1}<\theta_{N} \equiv \alpha$, and set $h_{i}$ : $=\theta_{i}-\theta_{i-1}, i=1,2, \ldots, N$. Let $\left\{L_{i}(\theta)\right\}$ be the system of linear interpolation functions on boundary $\Gamma$. It is easy

to know that $\sum_{i=0}^{N} L_{i}(\theta)=1$ and $S^{h}=\left\{L_{i}(\theta)\right\}_{i=0}^{N} \subset H^{\frac{1}{2}}(\Gamma)$. Then

$$
u_{0}^{h}(R, \theta)=\sum_{i=0}^{N} U_{i} \cdot L_{i}(\theta)
$$

Substituting (26) into (25), we easily obtain the system of linear algebraic equations of variational problem (25) as follows:

$$
Q \cdot U=b
$$

$$
\left[\begin{array}{cccccc}
q_{00} & q_{01} & q_{02} & \cdots & q_{0, N-1} & q_{0, N} \\
q_{10} & q_{11} & q_{12} & \cdots & q_{1, N-1} & q_{1, N} \\
q_{20} & q_{21} & q_{22} & \cdots & q_{2, N-1} & q_{2, N} \\
\vdots & \vdots & \vdots & \vdots & \vdots & \vdots \\
q_{N, 0} & q_{N, 1} & q_{N, 2} & \cdots & q_{N, N-1} & q_{N, N}
\end{array}\right]\left[\begin{array}{c}
U_{0} \\
U_{1} \\
U_{2} \\
\vdots \\
U_{N}
\end{array}\right]=\left[\begin{array}{c}
b_{0} \\
b_{1} \\
b_{2} \\
\vdots \\
b_{N}
\end{array}\right]
$$

where $Q:=\left(q_{i j}\right)_{(N+1) \times(N+1)}, q_{i j}:=\hat{D}\left(L_{i}(\theta), L_{j}(\theta)\right), U$ $:=\left(U_{0}, U_{1}, \ldots, U_{N}\right)^{T}, b:=\left(b_{0}, b_{1}, \ldots, b_{N}\right)^{T}, b_{i}:=$ $\hat{F}_{n}\left(L_{i}(\theta)\right) \equiv R \int_{0}^{\alpha} g_{n}(R, \theta) L_{i}(\theta) d \theta$. Using (15), it is not difficult to get that $q_{i j}$ are expressed as follows

$$
\begin{aligned}
& q_{i j}=q_{j i}=\frac{\pi}{\alpha^{2}} \sum_{n=0}^{+\infty} \varepsilon_{n} Z_{n} \cdot q_{i}(n) \cdot q_{j}(n), \\
& i, j=0,1,2, \ldots, N \\
& q_{k}(n)=\int_{0}^{\alpha} L_{k}(\theta) \cos \frac{n \pi \theta}{\alpha} d \theta, \quad k=0,1, \ldots, N .
\end{aligned}
$$

where

$$
\begin{aligned}
& q_{0}(n)=\left\{\begin{array}{l}
\frac{1}{2} h_{1}, n=0, \\
\frac{2 \alpha^{2}}{n^{2} \pi^{2} h_{1}} \sin ^{2}\left(\frac{n \pi \theta_{1}}{2 \alpha}\right),
\end{array} \quad n=1,2, \ldots\right. \\
& q_{N}(n)=\left\{\begin{array}{l}
\frac{1}{2} h_{N}, n=0, \\
-\frac{2 \alpha^{2}}{n^{2} \pi^{2} h_{N}} \sin \frac{n \pi\left(\theta_{N}+\theta_{N-1}\right)}{2 \alpha} \\
\cdot \sin \frac{n \pi\left(\theta_{N}-\theta_{N-1}\right)}{2 \alpha},
\end{array}\right. \\
& n=1,2, \ldots
\end{aligned}
$$

$$
q_{k}(n)=\left\{\begin{array}{l}
\frac{1}{2}\left(h_{k}+h_{k+1}\right), n=0, \\
-\frac{\alpha^{2}}{n^{2} \pi^{2}}\left\{\frac{1}{h_{k}} \cos \frac{n \pi \theta_{k-1}}{\alpha}\right. \\
-\left(\frac{1}{h_{k}}+\frac{1}{h_{k+1}}\right) \cos \frac{n \pi \theta_{k}}{\alpha} \\
+\frac{1}{h_{k+1}} \cos \frac{n \pi \theta_{k+1}}{\alpha}, n=1,2, \ldots
\end{array}\right.
$$

$k=1,2, \ldots, N-1$.

Especially, we partition boundary $\Gamma$ into uniform subdivision, that is $h=h_{i}=\frac{\alpha}{N}, \theta_{i}=i \bullet h \equiv i \bullet \frac{\alpha}{N}$, we now have 


$$
\begin{aligned}
& q_{0}(n)=\left\{\begin{array}{l}
\frac{\alpha}{2 N}, n=0, \\
\frac{2 \alpha N}{n^{2} \pi^{2}} \sin ^{2}\left(\frac{n \pi}{2 N}\right), n=1,2, \ldots
\end{array}\right. \\
& q_{N}(n)=\left\{\begin{array}{l}
\frac{\alpha}{2 N}, n=0, \\
(-1)^{n+2} \frac{2 \alpha N}{n^{2} \pi^{2}} \sin ^{2}\left(\frac{n \pi}{2 N}\right), n=1,2, \ldots
\end{array}\right. \\
& q_{k}(n)=\left\{\begin{array}{l}
\frac{\alpha}{N}, n=0, \\
-\frac{4 N \alpha}{n^{2} \pi^{2}} \sin ^{2}\left(\frac{n \pi}{2 N}\right) \\
\cdot \cos \left(\frac{k}{N} \cdot n \pi\right), n=1,2, \ldots
\end{array}\right. \\
& k=1,2, \ldots, N-1
\end{aligned}
$$

\section{Quadratic Element}

We now partition boundary $\Gamma$ into $2 N$ parts, the points of division are $\theta_{i}, i=1,2, \ldots, 2 N-1$, i.e., $0 \equiv \theta_{0}$ $<\theta_{1}<\theta_{2} \ldots<\theta_{i}<\theta_{i+1}<\ldots<\theta_{2 N-1}<\theta_{2 N} \equiv \alpha$, and set $h_{i}:=\theta_{i}-\theta_{i-1}, i=1,2, \ldots, 2 N$. Let $\left\{\varphi_{i}(\theta)\right\}$ be the system of quadratic interpolation functions on boundary $\Gamma$, it is easy to know that $\sum_{i=0}^{2 N} \varphi_{i}(\theta)=1$ and $S^{h}=\left\{\varphi_{i}(\theta)\right\}_{i=0}^{2 N} \subset$ $H^{\frac{1}{2}}(\Gamma)$. Similar to the case of linear boundary element, we easily obtain the system of linear algebraic equation of problem (25), which is similar to the case of linear boundary element, Equation (27). However, $Q=$ $\left(q_{i j}\right)_{(2 N+1) \times(2 N+1)}$, and $q_{i j}$ are given by the followings:

$$
\begin{aligned}
& q_{i j}=q_{j i}=\frac{\pi}{\alpha^{2}} \sum_{n=0}^{+\infty} \varepsilon_{n} Z_{n} \cdot q_{i}(n) \cdot q_{j}(n), \\
& i, j=0,1,2, \ldots, 2 N-1,2 N \\
& q_{k}(n)=\int_{0}^{\alpha} \varphi_{k}(\theta) \cos \frac{n \pi \theta}{\alpha} d \theta, \\
& k=0,1,2, \ldots, 2 N-1,2 N
\end{aligned}
$$

where

$$
q_{2 k-1}(n)=\left\{\begin{array}{l}
\frac{\left(h_{2 k}+h_{2 k-1}\right)^{3}}{6 h_{2 k} h_{2 k-1}}, n=0, \\
\left(\frac{\alpha}{n \pi}\right)^{2} \frac{1}{h_{2 k} h_{2 k-1}}\left\{\left(h_{2 k}\right.\right. \\
\left.+h_{2 k-1}\right)\left(\cos \frac{n \pi \theta_{2 k}}{\alpha}\right. \\
\left.+\cos \frac{n \pi \theta_{2 k-2}}{\alpha}\right) \\
-\frac{2 \alpha}{n \pi}\left(\sin \frac{n \pi \theta_{2 k}}{\alpha}\right. \\
\left.\left.-\sin \frac{n \pi \theta_{2 k-2}}{\alpha}\right)\right\}, \\
n=1,2, \ldots
\end{array}\right.
$$

$$
\begin{aligned}
k=1,2 & , \ldots, N \\
q_{2 k}(n)= & \frac{1-\delta_{0}^{k}}{h_{2 k}\left(h_{2 k}+h_{2 k-1}\right)} I_{k 1}(n) \\
& +\frac{1-\delta_{N}^{k}}{h_{2 k+1}\left(h_{2 k+1}+h_{2 k+2}\right)} I_{k 2}(n),
\end{aligned}
$$

$k=0,1,2, \ldots, N$

$$
I_{k 1}(n)=\left\{\begin{array}{c}
\frac{\left(h_{2 k}+h_{2 k-1}\right)^{2}\left(2 h_{2 k}-h_{2 k-1}\right)}{6}, n=0, \\
\frac{\alpha}{n \pi}\left\{h_{2 k}\left(h_{2 k}+h_{2 k-1}\right) \sin \frac{n \pi \theta_{2 k}}{\alpha}\right. \\
+\frac{\alpha}{n \pi}\left[\left(2 h_{2 k}+h_{2 k-1}\right) \cos \frac{n \pi \theta_{2 k}}{\alpha}\right. \\
+h_{2 k-1} \cos \frac{n \pi \theta_{2 k-2}}{\alpha}-\frac{2 \alpha}{n \pi} \\
\left.\left.+\left(\sin \frac{n \pi \theta_{2 k}}{\alpha}-\sin \frac{n \pi \theta_{2 k-2}}{\alpha}\right)\right]\right\}, \\
n=1,2, \ldots
\end{array}\right.
$$

$$
I_{k 2}(n)=\left\{\begin{array}{c}
\frac{\left(h_{2 k+1}+h_{2 k+2}\right)^{2}\left(2 h_{2 k+1}-h_{2 k+2}\right)}{6}, n=0, \\
\frac{\alpha}{n \pi}\left\{-h_{2 k+1}\left(h_{2 k+1}+h_{2 k+2}\right) \sin \frac{n \pi \theta_{2 k}}{\alpha}\right. \\
+\frac{\alpha}{n \pi}\left[\left(2 h_{2 k+1}+h_{2 k+2}\right) \cos \frac{n \pi \theta_{2 k}}{\alpha}\right. \\
+h_{2 k+2} \cos \frac{n \pi \theta_{2 k+2}-\frac{2 \alpha}{n \pi}}{\alpha} \\
\left.\left.\cdot\left(\sin \frac{n \pi \theta_{2 k+2}}{\alpha}-\sin \frac{n \pi \theta_{2 k}}{\alpha}\right)\right]\right\}, \\
n=1,2, \ldots .
\end{array}\right.
$$

\section{CONVERGENCE AND PRIORI ERROR ESTIMATES}

Let $u_{0}$ be the solution of natural integral equation (15), and $u_{0}^{h}$ be the corresponding solution of natural boundary element, which is the solution of problem $\left(P_{h}\right)$. The parameter $h$ is stated as in section 3 .

$\|\bullet\|_{\hat{D}}:=\sqrt{\hat{D}(\bullet, \bullet)}$. Let $\Gamma$ be the circular arc of boundary of domain $\Omega$ or $\Omega^{c}$. In this section we will present the convergence and error estimates on boundary $\Gamma$ for the numerical solution of the natural integral equation.

For the natural boundary element of elliptic boundary-value problems, under a united frame in Reference [10] has acquired some theorems with respect to the convergence and error estimates on boundary $\Gamma$ for the numerical solution of natural integral equation. Since 
the inferences of these theorems do not depend on the concrete expression of natural integral operator $\mathcal{K}$, so we can directly write out the following theorems.

Theorem 3. (convergence) The numerical solution $u_{0}^{h}$ obtained by natural boundary element converges to the exact solution $u_{0}$ in terms of energy norm $\|\bullet\|_{\hat{D}}$, that is

$$
\lim _{h \rightarrow 0}\left\|u_{0}-u_{0}^{h}\right\|_{\hat{D}}=0
$$

Theorem 4. (priori error estimates) If $u_{0} \in H^{r+1}(\Gamma)$, then the solution of natural integral equation has the following priori error estimates:

$$
\begin{aligned}
& \left\|u_{0}-u_{0}^{h}\right\|_{\hat{D}} \leq C h^{r+\frac{1}{2}}\left|u_{0}\right|_{r+1, \Gamma}, \quad(\text { energy norm })(44) \\
& \left\|u_{0}-u_{0}^{h}\right\|_{L^{2}(\Gamma)} \leq C h^{r+1}\left|u_{0}\right|_{r+1, \Gamma},\left(L^{2}-\right.\text { norm) } \\
& \left\|u_{0}-u_{0}^{h}\right\|_{L_{(\Gamma)}^{\infty}} \leq C h^{r+\frac{1}{2}}\left|u_{0}\right|_{r+1, \Gamma}, \\
& \text { (continuous norm) }
\end{aligned}
$$

where $r$ ( $r$ is a positive integer number) is the degree of piecewise interpolation polynomial, $C$ a positive constant independent of $u_{0}, h, \alpha$ and $R$. (45) and (46) hold only for $u_{0}$ satisfying $\int_{0}^{\alpha}\left[u_{0}(R, \theta)-u_{0}^{h}(R, \theta)\right] d \theta=0$, while $\beta=0$.

\section{NUMERICAL EXPERIMENTS}

In this section we only find the approximation $u_{0}^{h}(R, \theta)$ of $u_{0}(R, \theta)$ by using the natural boundary element method stated as the above. In fact, once the approximation $u_{0}^{h}(R, \theta)$ of $u_{0}(R, \theta)$ is obtained, the approximation $u^{h}(r, \theta)$ of the exact solution $u(r, \theta)$ in $\Omega$ or $\Omega^{c}$ can be acquired directly by Poisson integral formule. We here omit the part of computation. Since the elements of stiffness matrix $Q$ are given by some infinite series, we substitute $\sum_{n=1}^{M}$ into $\sum_{n=1}^{+\infty}$ in infinite series in practice computing the elements of stiffness matrix $Q$ (generally we take $M \leq 100$ ). The system of linear algebraic equations obtained by finite element discretization can be solved by Gauss-Seidel iteration method, and the number of iteration is denoted by $M_{1}$.

Table 1. Linear boundaey element (piecewise linear element), $R=1, \alpha=\frac{5 \pi}{4}$

\begin{tabular}{rrrccc}
\hline \multirow{2}{*}{$N$} & & & \multicolumn{3}{c}{$E_{\max }$} \\
\cline { 4 - 6 } & $M$ & $M_{1}$ & $\beta=-1$ & $\beta=0$ & $\beta=1$ \\
\hline 8 & 20 & 20 & $1.55 E-2$ & $2.11 E-2$ & $1.68 E-2$ \\
16 & 40 & 60 & $3.96 E-3$ & $5.56 E-3$ & $4.32 E-3$ \\
32 & 80 & 100 & $1.03 E-3$ & $1.43 E-3$ & $1.12 E-3$ \\
\hline
\end{tabular}

Denote the maxmal error of $L^{2}$-norm on boundary by $E_{\text {max }}$. To sure the problem (1)-(5) has an unique solution, some conditions must be added for $\beta=0 . U_{\left[\frac{N}{2}\right]}$ is taken to be zero while linear boundary element is used, and $U_{N}$ to be zero while quadratic boundary element is used.

Example 1. Solving the problem in exterior domain $\Omega^{c}$, where $R=1, \alpha=\frac{5 \pi}{4}$, and function $g_{n}(R, \theta)$ is as follows

$$
g_{n}(R, \theta)= \begin{cases}K_{\frac{4}{5}}^{\prime}(R) \cdot \cos \frac{4 \theta}{5}, & \beta=-1 ; \\ \cos \frac{4 \theta}{5}, & \beta=0 ; \\ H_{\frac{4}{5}}^{(1)^{\prime}}(R) \cdot \cos \frac{4 \theta}{5}, & \beta=1 .\end{cases}
$$

This problem has the exact solution:

$$
u(r, \theta)= \begin{cases}-K_{\frac{4}{5}}(r) \cdot \cos \frac{4 \theta}{5}, & \beta=-1 ; \\ \frac{5}{4} r^{-\frac{4}{5}} \cos \frac{4 \theta}{5}, & \beta=0 \\ -H_{\frac{4}{5}}^{(1)}(r) \cdot \cos \frac{4 \theta}{5}, & \beta=1\end{cases}
$$

where $r>R$ and $0<\theta<\alpha$. Numerical results are shown by Tables 1 and 2, respectively.

Example 2. Solving the problems in interior domain $\Omega$, where $R=1, \alpha=\frac{5 \pi}{4}$, and function $g_{n}(R, \theta)$ is as follows

$$
g_{n}(R, \theta)= \begin{cases}I_{\frac{4}{5}}^{\prime}(R) \cdot \cos \frac{4 \theta}{5}, & \beta=-1 ; \\ \cos \frac{4 \theta}{5}, & \beta=0 ; \\ J_{\frac{4}{5}}^{\prime}(R) \cdot \cos \frac{4 \theta}{5}, & \beta=1 .\end{cases}
$$

Now the exact solution of the problem is

$$
u(r, \theta)= \begin{cases}I_{\frac{4}{5}}(r) \cdot \cos \frac{4 \theta}{5}, & \beta=-1 ; \\ \frac{5}{4} r \frac{4}{5} \cos \frac{4 \theta}{5}, & \beta=0 ; \\ J_{\frac{4}{5}}(r) \cdot \cos \frac{4 \theta}{5}, & \beta=1 .\end{cases}
$$

where $r<R$ and $0<\theta<\alpha$. Numerical results are shown by Tables 3 and 4 , respectively.

Table 2. Quadratic boundary element (piecewise quadratic element), $R=1, \alpha=\frac{5 \pi}{4}$

\begin{tabular}{rrrccc}
\hline \multirow{2}{*}{$N$} & & & \multicolumn{3}{c}{$E_{\max }$} \\
\cline { 4 - 6 } & $M$ & $M_{1}$ & $\beta=-1$ & $\beta=0$ & $\beta=1$ \\
\hline 8 & 20 & 20 & $1.24 E-2$ & $2.53 E-2$ & $1.41 E-2$ \\
16 & 40 & 60 & $1.64 E-3$ & $2.41 E-3$ & $1.84 E-3$ \\
32 & 80 & 100 & $2.10 E-4$ & $3.07 E-4$ & $2.34 E-4$ \\
\hline
\end{tabular}


Table 3. Linear boundaey element (piecewise linear element), $R=1, \alpha=\frac{5 \pi}{4}$

\begin{tabular}{rrrccc}
\hline & & & \multicolumn{3}{c}{$E_{\max }$} \\
\cline { 4 - 6 }$N$ & $M$ & $M_{1}$ & $\beta=-1$ & $\beta=0$ & $\beta=1$ \\
\hline 8 & 20 & 20 & $1.69 E-2$ & $2.34 E-2$ & $1.75 E-2$ \\
16 & 40 & 60 & $4.34 E-3$ & $5.93 E-3$ & $4.47 E-3$ \\
32 & 80 & 100 & $1.13 E-3$ & $1.54 E-3$ & $1.15 E-3$ \\
\hline
\end{tabular}

\section{SOME APPLICATIONS}

Let $\Omega^{c}$ be an unbounded domain whose boundary is composed of two sides $\Gamma_{0}$ and $\Gamma_{\alpha}$ of a concave angle $0<\alpha<2 \pi$ and a simple curve $\Gamma$, i.e. $\Gamma$ is defined by any single-valued continuous function of $\theta, \theta \in(0, \alpha), \Gamma=$ $\{(r, \theta) \mid r=r(\theta), \theta \in(0, \alpha)\}$ and $\mathrm{r}=\mathrm{r}(\theta)$ is a single valued function. Considering the following value problem:

$$
\begin{cases}\Delta u+\beta u=0 & \text { in } \Omega^{c} \\ \frac{\partial u}{\partial n}(r, 0)=0, & \text { on } \Gamma_{0} \\ \frac{\partial u}{\partial n}(r, \alpha)=0, & \text { on } \Gamma_{\alpha} \\ \frac{\partial u}{\partial n}(r, \theta)=g_{n}(r, \theta), & \text { on } \Gamma_{\alpha} \\ \text { conditionsat infinity } & \end{cases}
$$

In $\Omega^{c}$ we draw a circlur arc $\Gamma_{R}=\{(R, \theta) \mid 0 \leq \theta \leq \alpha\}$, it divides $\Omega^{c}$ into $\Omega_{1}$ and $\Omega_{2}$. $\Omega_{2}$ is an infinite sector. It is not difficult to get that (47) is equivalent to

$$
\left\{\begin{array}{l}
\text { Find } u \in H^{1}\left(\Omega_{1}\right) \text { such that for any } v \in H^{1}\left(\Omega_{1}\right) \\
D_{1}(u, v)+\hat{D}_{2}(u, v)=\left\langle g_{n}, v>\Gamma\right.
\end{array}\right.
$$

where $D_{1}(u, v)=\int_{\Omega_{1}}(\nabla u \cdot \nabla v-\beta u v) d x, \hat{D}_{2}(u, v)=\int_{\Gamma_{R}} v$ $\cdot(\mathcal{K} u) d S \equiv R \int_{0}^{\alpha} v \cdot(\mathcal{K} u) d \theta,\left\langle g_{n}, v>\Gamma=\int_{\Gamma} g_{n} \cdot v d S\right.$.

$$
\begin{aligned}
\mathcal{K} u(R, \theta)= & \frac{\pi}{R \alpha^{2}} \sum_{n=0}^{+\infty} \varepsilon_{n} Z_{n} \int_{0}^{\alpha} v\left(R, \theta^{\prime}\right) \\
& \cdot \cos \frac{n \pi \theta}{\alpha} \cdot \cos \frac{n \pi \theta^{\prime}}{\alpha} d \theta^{\prime}
\end{aligned}
$$

Now we use the finite element method in $\Omega_{1}$. Set $V=\left(U_{0}, U_{1}, \ldots, U_{N}\right)^{T}$ is set of values at nodes on $\Gamma_{R}, U$ $=\left(U_{N+1}, U_{N+2}, \ldots, U_{N+M}\right)^{T}$ is set of values at nodes on $\Gamma$ and at interior nodes. $\left\{L_{i}(x, y)\right\}_{i=0}^{M+N} \subset H^{1}\left(\Omega_{1}\right)$ are corresponding basis function, fox example, piecewise linear, then restiction of $\Gamma_{R}$ are approximately piece wise linear on $\Gamma_{R}$. Let $u \approx \sum_{i=0}^{N+M} U_{i} \cdot L_{i}(x, y)$, we obtain

$$
\sum_{j=0}^{N+M} D_{1}\left(L_{i}, L_{j}\right) U_{j}+\sum_{j=0}^{N} \hat{D}_{2}\left(L_{i}, L_{j}\right) U_{j}
$$

Table 4. Quadratic boundary element (piecewise quadratic element), $R=1, \alpha=\frac{5 \pi}{4}$

\begin{tabular}{rrrccc}
\hline & & & \multicolumn{3}{c}{$E_{\max }$} \\
\cline { 4 - 6 }$N$ & $M$ & $M_{1}$ & $\beta=-1$ & $\beta=0$ & $\beta=1$ \\
\hline 8 & 20 & 20 & $1.32 E-2$ & $2.12 E-2$ & $1.69 E-2$ \\
16 & 40 & 60 & $1.68 E-3$ & $2.69 E-3$ & $2.14 E-3$ \\
32 & 80 & 100 & $2.13 E-4$ & $3.39 E-4$ & $2.71 E-4$ \\
\hline
\end{tabular}

$$
=\left\langle g_{n}, L_{i}\right\rangle_{\Gamma}, \quad i=0,1, \ldots, M+N
$$

namely

$$
\left[K+\left(\begin{array}{cc}
Q & 0 \\
0 & 0_{M \times M}
\end{array}\right)\right]\left[\begin{array}{c}
V \\
U
\end{array}\right]=\left[\begin{array}{c}
b_{0} \\
b_{1} \\
\vdots \\
b_{M+N}
\end{array}\right]
$$

where $K=\left(D_{1}\left(L_{i}, L_{j}\right)\right)_{(M+N+1) \times(M+N+1)}, Q=\left(\hat{D}_{2}\left(L_{i}, L_{j}\right)\right)$ $(N+1) \times(N+1), b_{i}=\left\langle g_{n}, L_{i}\right\rangle \Gamma . K$ can be obtained by FEM, $Q$ is given by above expreeions in Section 3 .

\section{CONCLUDING REMARKS}

We have derived a sequence of natural integral equations for solving elliptic problems with concave angle domains, including problems in interior domain and exterior domain in plane. A finite element formulation is presented in computing natural integral equations. Error estimates for finite element approximation are given, which depend on the parameter $h$. From our numerical results, we can make several concluding remarks:

1. It takes much time to obtain the numerical integration by using classical boundary element method, especially to deal with singular integral. However, for natural boundary element method we have seen that the explicit expressions of these elements of boundary element stiffness matrix are given (see Eqs. (29)(33) or (37)-(42) ). And they have some distinctive properties. It is easy to be implementated on calculation and storage comparing with classical boundary element methods.

2. To solve natural integral equation is very simple and easy in programming. At the same time, natural integral equation can be used as the artificial boundary condition in practice, and one can get better accuracy for solving problems in unbounded domains by standard finite element methods. Thus we recommend engineers to use the method.

3. Some domain decomposition methods based on natural boundary reduction have been used to solve some elliptic problems with unbounded domains or 
singularities. We shall report on progress in some of these directions in a future publication.

\section{ACKNOWLEDGMENT}

The authors first wish to thank the referees for many valuable sugguestions. Second, this work is partly supported by the Special Funds for State Major Basic Research Projects of China, Grant number 1999032800, and by the knowledge innovation program of the Chinese Academy Sciences.

\section{REFERENCES}

1. Yu, D. H.,"Harmonic Canonical Integral Equations with Cracked and Concave Angle Domains and Their Numerical Solutions", Journal of Numerical Methods and Computer Applications, Vol. 4, No. 3, pp. 193-198 (1983).

2. Feng, K.,"Finte Element Method and Natural Boundary Reduction", Proceeding of the International Congress of Mathematics, Warsawa, Polish Academy of Sciences, pp. 1439-1453 (1983).

3. Feng, K., "Asymptotic Radiation Conditions for Reduced Wave Equation", J. Comput. Math., Vol. 2, No. 2, pp. 130-136 (1984).

4. Feng, K. and Yu, D., "Canonical Integral Equations of Elliptic Boundary Value Problems and Their Numerical Solutions", Proceeding of China-France Symposium on Finite Element Method (1982, Beijing) Science Press (eds. Gordon and Breach), Beijing, pp. 211-252 (1983).

5. Keller, J. B. and Givoli, D., "Exact Nonreflecting Boundary Conditions", J. Comput. Phys., Vol. 82, No. 1, pp. 172-192 (1989).

6. Givoli, D. and Keller, J. B., "A Finite Element Method for Large Domain", Comput. Methods Appl. Mech. Eng., Vol. 76, No. 1, pp. 41-66 (1989).

7. Du, Q. K. and Yu, D. H., "On the Natural Integral Equation for Initial Boundary Value Problems of Two Dimensional Hyperbolic Equation", Acta Mathematica Applied Sinica, Vol. 24, No. 1, pp. 17-26 (2001).

8. Liu, S. K. and Liu, S. D., Special Functions, Atmospheric Phenomena Press, Beijing, (1988)(in Chinese).

9. Wang Z. X. and Guo, D. R., Introduction to Special Function, Peking University Press, Beijing, (2000)(in
Chinese).

10. Yu, D. H., Mathematical Theory of Natural Boundary Element Methods, Science Press, Beijing, (1993)(in Chinese).

11. Givoli, D., Rivkin, L. and Keller, J. B., "A finite element method for domains with corners", Int. J. Numer. Meth. Engng., Vol. 35, pp. 1329-1345 (1992).

12. Wu, X. and Han, H., "A finite-element method for Laplace and Helmholtz-type boundary value problems with singularities", SIAM J. Numer. Anal., Vol. 34, No. 3, pp. 1037-1050 (1997).

13. Bao, W. Z. and Han, H., "High-order local artificial boundary conditions for problems in unbounded domains", Comput. Methods Appl. Mech. Eng.," Vol. 188, No. 1/3, pp. 455-471 (2000).

\section{凹角型區域橢圆邊值問題的自然邊 界元法

$$
\text { 杜 其 奎 }
$$

南京師範大學數學與計算機科學學院

$$
\text { 余 德 浩 }
$$

中國科學院數學與系統科學研究院 計算數學與科學工程算研究所

$$
\text { 摘 要 }
$$

本文研究凹角型區域楕圆邊值問題的自然邊界 歸化及其邊界元法。給出了自然總分方程及相應的 Poisson總分公式與近似解的收斂性及誤差估計, 詳 細地討論了自然總分方程的有限元數值方法, 我們給 出了數值試驗以説明方法的有效性。作爲應用, 我們 也研究了無限扇形區域的有限元與自然邊界元耦合 法。

關鍵詞：自然邊界歸化; 凹角區域; 數值方法; 耦 合。 